\title{
In Doppelblindstudien sehr viel seltener als im klinischen Alltag
}

Fragestellung: Wie häufig treten Muskelbeschwerden auf, wenn die Einnahme eines Statins verblindet oder offen erfolgt?

Hintergrund: In den letzten 20 Jahren hat eine Vielzahl von Studien belegt, dass insbesondere bei Patienten mit erhöhten Serum-Cholesterinspiegeln die Einnahme von Statinen das Risiko von Herzinfarkten und ischämischen Insulten reduziert. Die am häufigsten geklagte Nebenwirkung von Statinen sind Muskelschmerzen. Dies führt bei einem nicht unerheblichen Prozentsatz der Patienten zum Therapieabbruch. Die Frage, ob immer ein kausaler Zusammenhang zwischen der Einnahme eines Statins und Muskelschmerzen besteht, kann im klinischen Alltag allerdings nur dadurch beantwortet werden, indem das Statin pausiert wird. Ob die Kenntnis darüber, dass ein Statin eingenommen wird oder nicht, die Häufigkeit von Muskelbeschwerden bestimmt, wurde in einer Sekundäranalyse der ASCOT-LLA-Studie untersucht.

Gupta A, Thompson D, Whitehouse $A$ et al. Adverse events associated with unblinded, but not with blinded, statin therapy in the Anglo-Scandinavian Cardiac Outcomes Trial-Lipid-Lowering Arm (ASCOT-LLA): a randomised double-blind placebo-controlled trial and its non-randomised non-blind extension phase. Lancet 2017; 389: $2473-81$
Patienten und Methodik: Die ASCOT-LLA-Studie wurde in England und Skandinavien zwischen 1998 und 2002 durchgeführt. Eingeschlossen wurden Personen mit multiplen Risikofaktoren für kardiovaskuläre Erkrankungen ohne einen stattgehabten Myokardinfarkt. In einem $2 \times 2$ faktori- ellen Design wurden zwei Antihypertensivaschemata mit einem Kalziumantagonisten beziehungsweise einem Betablocker untersucht und im zweiten Studienarm $10 \mathrm{mg}$ Atorvastatin mit Placebo verglichen. In den doppelblinden Teil der Studie wurden 10.180 Patienten eingeschlossen und im Median über drei Jahre verfolgt. Die Studie wurde vorzeitig abgebrochen, nachdem eine Zwischenanalyse eine signifikante Überlegenheit des Statins gezeigt hatte. Den Patienten wurde dann angeboten, über drei weitere Jahre mit Atorvastatin offen behandelt zu werden. In dem entsprechenden Zeitraum zwischen 2002 und 2005 entschlossen sich 6.409 Patienten (65\%), Atorvastatin einzunehmen, und 3.490 (35\%) verzichteten auf die Einnahme von Atorvastatin. In der doppelblinden Studienphase waren Muskelschmerzen, die als unerwünschte Ereignisse gemeldet wurden, mit 2,03\% und 2,0 \% zwischen Atorvastatin und Placebo nicht unterschiedlich. In der offenen Behandlungsphase wurden muskelbezogene Nebenwirkungen um $40 \%$ häufiger unter dem Statin als unter Placebo berichtet. Die Hazard Ratio von 1,41 war mit einem p-Wert von 0,006 signifikant. Für andere Nebenwirkungen zeigten sich keine Unterschiede zwischen den Patienten, die das Statin einnahmen, und denjenigen, die kein Statin anwendeten.

Schlussfolgerungen: In einer großen Behandlungsstudie, die in der ersten Hälfte doppelblind und in der zweiten Hälfte offen durchgeführt wurde, zeigte sich, dass muskelbezogene Nebenwirkungen unter der Einnahme von Statinen signifikant häufiger sind, wenn Patienten und Arzt wissen, dass die Behandlung mit einem Statin erfolgt.

\section{Gratwanderung zwischen Aufklärung und Noceboeffekt-Provokation}

Die Post-hoc-Analyse der ASCOT-LLA-Studie ist ein gutes Beispiel für den Noceboeffekt von Medikamenten. Während in der verblindeten Phase der Studie kein Unterschied zwi-

Weitere Infos auf springermedizin.de

ESC/EAS-Leitlinien zur Behandlung von Dyslipidämien

Die europäischen Leitlinien betonen das LDL-Cholesterin als das wichtigste Behandlungsziel, nennen bevölkerungsbezogene Präventionsmaßnahmen und behandeln detailliert die Diagnostik und Therapie genetischer Dyslipidämien. Diesen Artikel finden Sie, indem Sie den Titel in die Suche eingeben. schen muskelbezogenen Nebenwirkungen einer niedrigen Dosis von Atorvastatin bestand, fand sich in der offenen Behandlungsphase eine signifikante Erhöhung der Häufigkeit dieser Nebenwirkungen. Ärzte sind verpflichtet, Patienten die an einer klinischen Studie teilnehmen, über potenzielle Nebenwirkungen der Studienmedikation aufzuklären. Die Häufigkeit der Nebenwirkungsmeldungen hängt davon ab, ob spontane Meldungen von Patienten berücksichtigt oder ob potenzielle Nebenwirkungen gezielt abgefragt werden. Im klinischen Alltag ist es außerordentlich schwierig, eine gute Balance zwischen der ethisch und rechtlich notwendigen Aufklärung von Patienten über potenzielle Nebenwirkungen und der Provokation von Noceboeffekten zu finden. In modernen Studien werden Studienärzte und Patienten gezielt geschult, um den Nocebo- und Placeboeffekt so gering wie möglich zu halten. 